\title{
Whole-cell antigenicity data support sequence-based kinetoplastid taxonomy
}

\author{
Bernard Couvreur ${ }^{+}$ \\ ASBL Culture in vivo, Nivelles, Belgium
}

Early immunological data, obtained by immunodiffusion and immunoelectrophoresis, on the whole-cell antigenicity of kinetoplastid protozoa were retrieved and used to construct a dendrogram of antigenic distances. Remarkably, they supported the same taxonomic conclusions as analyses based on DNA and protein sequence data.

Key words: molecular taxonomy - Leishmania - Trypanosoma - antigen - immunodiffusion

The taxonomy and evolutionary history of kinetoplastids has long been of great interest to parasitologists because of the biological interest of the topic and the medical importance of certain members of this order (reviewed by Simpson et al. 2006). Recent debate has focused on the relationships between African and American trypanosomes. Some authors have criticised phylogenetic studies based on rRNA sequences and have brought arguments against the validity of the genus Trypanosoma (e.g., Piontkivska \& Hughes 2005). Others, including Simpson et al. (2006), have considered the existence of this genus to be robustly supported by ribosomal and protein sequence data. This question has recently been readdressed by Leonard et al. (2011) using whole-genome datasets of protein-coding genes and improved phylogenetic methodologies. Other issues in kinetoplastid taxonomy include the relationships between trypanosomatids and free-living kinetoplastids, the relationships among species within the genera Trypanosoma and Leishmania and the position of lizard Leishmania species (Simpson et al. 2006, Stevens 2008, Fraga et al. 2010).

Remarkably, even though they focused on a small number of medically important species, the findings of several studies from the 1970s using precipitation-in-gel techniques [immunodiffusion and immunoelectrophoresis (IEP)] support the findings of later molecular studies. Using the methodology pioneered by Capron (1970), Le Ray (1975), Afchain (1976) and Afchain et al. (1979) explored the antigenic make-up of medically important trypanosomatids and compared these trypanosomatids with more distantly related microorganisms.

This approach has several interesting features. First, it can be used to analyse all types of antigenic molecules - not only polypeptides, but also post-translationally

+ Corresponding author: couvreur.bernard@gmail.com Received 16 September 2012

Accepted 23 November 2012 modified proteins, protein complexes, as well as lipids and glycolipids. Second, precipitation-in-gel techniques produce minimal denaturation. Accordingly, antigens that would likely have been overlooked by techniques such as western blotting or phage library screening can be identified. In essence, microorganisms are compared on the basis of their complete collection of antigenic determinants, allowing the rabbit immune system to decide which antigens are identical and which are different.

The authors of the cited early papers repeatedly immunised rabbits with extracts of the free-living forms of well-characterised strains of kinetoplastid protozoa grown under axenic conditions. These researchers monitored the immune response by IEP, counting the number of precipitation lines that appeared when the sera were tested against homologous extracts. Over the immunisation period, the number of lines steadily increased and eventually stabilised at 30-35, depending on the protozoon species being investigated. The relationships between species could then be investigated experimentally by testing these hyperimmune sera against soluble extracts from increasingly distantly related organisms, starting with the components of the culture medium. Hyperimmune sera were also adsorbed with heterologous extracts and tested against the homologous (immunising) extract to crosscheck "shared" lines. All experiments were repeated in two separate institutions (i.e., the Pasteur Institute in Lille, France, and Prince Leopold Institute for Tropical Medicine in Antwerp, Belgium). The authors recorded the number of cross-reacting lines, i.e., antigens shared by two organisms and went on to calculate an "isology index" (I) as the number of lines shared by two organisms on the total number of lines for a given hyperimmune serum. These authors could then propose taxonomic relationships based on molecular data in a quantitative manner, although no bootstrap values could be calculated, making it difficult to assess the robustness of the reconstruction. A numerical analysis of the collective data of Le Ray (1975), Afchain (1976) and Afchain et al. (1979) is presented in Figure. The I was transformed into an "antigenic distance" (AD) using the equation $\mathrm{AD}=1-\mathrm{I}$. The relationships and $\mathrm{ADs}$ were written using the "Newick" format with parentheses 


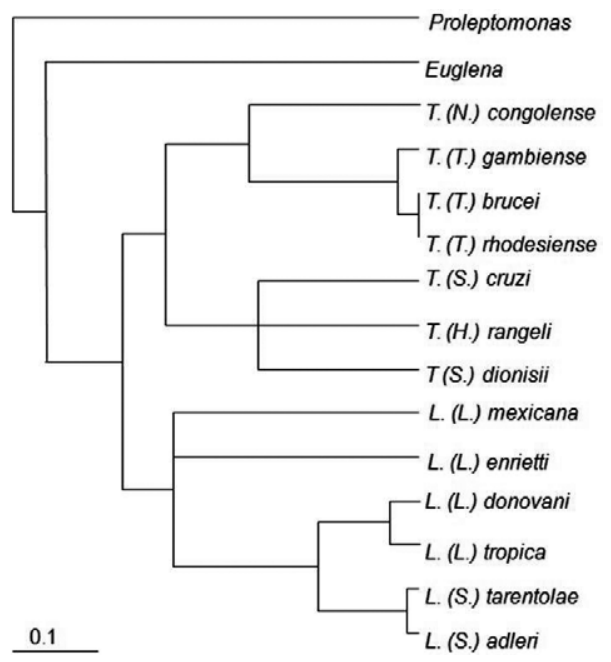

Relationships between trypanosomatids as deduced from antigenicity data. Data were retrieved from Le Ray (1975), Afchain (1976) and Afchain et al. (1979). Branch lengths are proportional to antigenic distance $=1-$ number shared immunoelectrophoresis (IEP) lines/total number of IEP lines]. Nodes were placed arbitrarily at mid-lengths. The scale is given by the score bar at the lower left of the picture. Data were written in the parenthesed "newick" format and introduced into program TreeView (Page 1996) to redraw the dendrogram. The major conclusions are consistent with sequence-based taxonomy.

to express the $\mathrm{AD}$ values as branch lengths. This formula was introduced into the TreeView program (Page 1996) to generate the dendrogram in Figure.

The following observations could be made: (i) there are specific relationships between trypanosomatids and euglenids (i.e., supporting the taxonomic group Euglenozoa), (ii) there are specific relationships between American and African trypanosomes, excluding Leishmania (consistent with a bona fide status for both the genus Trypanosoma and the genus Leishmania), (iii) there are close relationships between the reptile parasites Leishmania (S.) tarentolae and Leishmania (S.) adleri with Leishmania (L.) donovani and Leishmania (L.) tropica within the genus Leishmania, (iv) Trypanosoma (S.) cruzi groups with Trypanosoma (H.) rangeli and Trypanosoma (S.) dionisii away from the group consisting of Trypanosoma (N.) congolense, Trypanosoma (T.) gambiense, Trypanosoma (T.) brucei and Trypanosoma (T.) rhodesiense (supporting the existence of a "T. cruzi" clade and a "T. brucei" clade) and (v) T. (T.) brucei and $T$. (T.) rhodesiense are antigenically identical.

The existence of the taxonomic group Euglenozoa and the monophyly of the genus Trypanosoma are now considered resolved issues (Simpson et al. 2006, Stevens 2008, Leonard et al. 2011). The existence of a "T. cruzi" clade and a "T. brucei" clade is also well supported by phylogenetic analyses of their GAPDH and small subunit rRNA coding sequences (Stevens et al. 2001, Hamilton et al. 2004, Stevens 2008). So is the close relatedness of T. (T.) brucei and T. (T.) rhodesiense (Stevens et al. 2001, Hamilton et al. 2004, Stevens 2008). The ADs are also consistent with the reptile parasites $L$. (S.) tarentolae and $L$. (S.) adleri and the mammalian leishmanias forming a monophyletic group. This conclusion is consistent with the most recent molecular studies on the subject, which were based on $h s p 70$ sequences (Fraga et al. 2010). However, the close antigenic proximity of $L$. (S.) tarentolae and $L$. (S.) adleri to Old World Leishmania within the subgenus $L$. (Leishmania) does not support the existence of the subgenus L. (Sauroleishmania).

Nevertheless, this dendrogram has its peculiarity. Proleptomonas would be expected to branch off near Trypanosoma and Leishmania or, at least, within the Euglena-Trypanosoma group (aka Euglenozoa) because Proleptomonas was initially considered a free-living trypanosomatid (Le Ray 1975, Vickerman et al. 2002). However, it has very few antigens in common with trypanosomes and Leishmania and it branches at the bottom at the dendrogram in Figure. More recently, the re-evaluation of morphological and molecular data has shown Proleptomonas to be a relative of the cercomonads (Vickerman et al. 2002). The placement of Proleptomonas as an outgroup with respect to Euglenozoa is thus consistent with modern taxonomical conceptions.

We conclude that the most recent views of kinetoplastid taxonomy are supported by antigenicity data in addition to protein and DNA sequence data. Other conclusions from early works on the molecular biology of trypanosomatids, including specific antigens proposed for diagnosis and/or vaccination, are probably worth reconsidering too.

\section{ACKNOWLEDGEMENTS}

To Dr Alexandra Briquet and Dr Catherine DedoyartMenten, Groupe Interdisciplinaire de Génoprotéomique Appliquée, Université de Liège, Belgium, for their assistance in preparing the Figure.

\section{REFERENCES}

Afchain D 1976. Le caractère antigénique des Trypanosomatidae hétéroxènes parasites de l'homme. Trypanosoma (S.) cruzi, Trypanosoma (T.), B. gambiense et Leishmania donovani, PhD Thesis, Université des Sciences et Techniques, Lille, 56 pp.

Afchain D, Le Ray D, Fruit J, Capron A 1979. Antigenic make-up of Trypanosoma cruzi culture forms: identification of a specific component. J Parasitol 65: 507-514.

Capron AR 1970. Parasitic antigen. Structure and function. J Parasitol 56: 515-521.

Fraga J, Montalvo AM, de Doncker S, Dujardin JC, Van der Auwera G 2010. Phylogeny of Leishmania species based on the heat-shock protein 70 gene. Infect Genet Evol 10: 238-245.

Hamilton PB, Stevens JR, Gaunt MW, Gidley J, Gibson WC 2004. Trypanosomes are monophyletic: evidence from genes for glyceraldehyde phosphate dehydrogenase and small subunit ribosomal RNA. Int J Parasitol 34: 1393-1404.

Le Ray D 1975. Antigenic structure of Trypanosoma brucei (protozoa, kinetoplastids). Immunoelectrophoretic analysis and comparative study. Ann Soc Belg Med Trop 55: 129-311.

Leonard G, Soanes DM, Stevens JR 2011. Resolving the question of trypanosome monophyly: a comparative genomics approach using whole genome data set with low taxon sampling. Infect Genet Evol 11: 955-959. 
Page RDM 1996. TreeView: an application to display phylogenetic trees on personal computers. Comput Appl Biosci 12: 357-358.

Piontkivska H, Hughes AL 2005. Environmental kinetoplastid-like $18 \mathrm{~S}$ rRNA sequences and phylogenetic relationships among Trypanosomatidae: paraphyly of the genus Trypanosoma. Mol Biochem Parasitol 144: 94-99.

Simpson AG, Stevens JR, Lukes J 2006. The evolution and diversity of kinetoplastid flagellates. Trends Parasitol 22: 168-174.
Stevens JR 2008. Kinetoplastid phylogenetics with special reference to the evolution of parasitic trypanosomes. Parasite 5: 226-232.

Stevens JR, Noyes HA, Schofield CJ, Gibson W 2001. The molecular evolution of Trypanosomatidae. Adv Parasitol 48: 1-56.

Vickerman K, Le Ray D, Hoef-Emden K, de Jonckheere J 2002. The soil flagellate Proleptomonas faecicola: cell organisation and phylogeny suggest that the only described free-living trypanosomatid is not a kinetoplastid but has cercomonad affinities. Protist 153: 9-24. 